\title{
Programa de economía de fichas en el hogar*
}

\section{A home-based token economy program}

\author{
Franklin Soler ${ }^{* *}$ \\ Resumen \\ Johanna Paola Herrera \\ Sebastián Buitrago \\ Linney Barón \\ Universidad Santo Tomás, \\ Bogotá, Colombia \\ Se aplica un programa de economía de fichas para \\ modificar algunos comportamientos disruptivos de \\ un niño. Se pretende decrementar el contestar de \\ manera inadecuada, así como hacer pataletas y pro- \\ piciar la ocurrencia del seguimiento de instrucciones \\ relacionadas con conductas como arreglar la cama, \\ recoger y organizar los juguetes, recoger y organi- \\ zar la ropa, hacer sus deberes escolares. Se hace una \\ profunda revisión de la aplicación del programa de \\ Economía de Fichas en diversos campos y problemas \\ de conducta. Siguiendo un diseño ABA, los resultados \\ Recibido: 2 de febrero de 2009 \\ confirman el cambio de comportamiento y logran un \\ Revisado: 28 de marzo de 2009 \\ mejor ajuste de la conducta del niño en la escuela y \\ Aceptado: 29 de mayo de 2009 \\ Correspondencia: Franklin Soler, Facultad de Psicología. Universidad Santo Tomás. Correo electrónico:fgsoler@gmail.com. Dirección postal: Autopista \\ Norte, calle 209, Vía Arrayanes, Km 1.5, Bogotá, Colombia.
}

Artículo de investigación. 


\section{Abstract}

It applies a token economy program to modify some disruptive behaviors of a child. It is intended to decrease the answer in an inappropriate manner and make tantrums and encourage the occurrence of follow-up instructions for behaviors such as to fix the bed, collecting and organizing toys, collect and organize your clothes, do their homework. It is a thorough review of the implementation of the token economy in various fields and behavioral problems. Following an ABA design, the results confirm the behavior change made a better adjustment of the child's behavior at school and home environment. An analysis is made from some of the principles of behavioral economics.

Keywords: token economy, behavior modification, enhancement, economic balance, desirable behavior, undesirable behavior.

\section{Introducción}

La Economía de Fichas EF puede considerarse como un sistema motivacional que se aplica con el fin de modificar conductas específicas en una persona y mejorar su adaptación al medio ambiente (Alvord, 1974). Uno de los aspectos más interesantes del programa es el sistema de reforzamiento que utiliza, en el cual la ocurrencia de conductas apropiadas o la ausencia de conductas problemáticas se mantiene por refuerzo secundario a través de fichas durables, que pueden ser periódicamente cambiadas por otros reforzadores.

Montrose Wolf, primer editor del Journal of Applied Bahavior Analysis es quizás quién ha consolidado la economía de fichas como un plan de intervención conductual, aunque se han reseñado trabajos pioneros desde el siglo XIX en los que se dieron lineamientos para establecer este sistema y fomentar la disciplina en clase (Olivares, Montesinos \& Preciado, 2005).

Quizás los dos grandes pioneros del sistema de economía de fichas aplicado en la modificación de conductas a nivel institucional de manera sistematizada, organizada y con un fuerte sustento en los principios del análisis experimental del comportamiento fueron Ayllon \& Azrin (1974), quienes hicieron recomendaciones para una aplicación adecuada del procedimiento, a partir de su experiencia en un hospital psiquiátrico. Propusieron que las conductas a modificar deben describirse de manera clara y precisa, evitando interpreta- ciones variadas, al igual que se debe hacer un seguimiento sistemático del modo de vida del sujeto, sus costumbres, rutinas y así seleccionar los reforzadores que respondan a las necesidades más vitales del sujeto, garantizando que los efectos del programa no estén relacionados con las características de los administradores de los tratamientos, sino que se centren en los eventos reforzantes. Al respecto se debe tener capacidad y autonomía para responder a las solicitudes que haga el sujeto, esto puede aportar al tratamiento al conocerse nuevos reforzadores de su parte. Para desarrollar una cadena de respuestas deseadas, es necesario empezar reforzando una respuesta existente relacionada con la conducta terminal, aspecto que refiere a la conocida regla del moldeamiento. También es necesario utilizar un lenguaje claro, constante (sencillo y sin tecnicismos); los registros y avances se deben expresar cuantitativamente y permitir a otros experimentadores conocer los resultados obtenidos para recibir sugerencias o posteriores replicaciones.

En cuanto a la entrega de reforzadores en el sistema, se debe tener presente que si un reforzador es efectivo y agrega valor al tratamiento, las variaciones que éste pueda tener constituyen también un valor a tener en cuenta. Como es posible que se presente saciedad frente a un reforzador, no debería usarse nunca un solo evento como reforzador sino tener una amplia gama de posibilidades. En este sentido, cuando se programan simultáneamente dos o más reforzadores, a menudo los pacientes seleccionan uno y excluyen los demás. Es necesario anticiparse a la posible 
saciedad con el reforzador: a mayor número de fichas otorgadas por la ejecución de la conducta deseada, más frecuente será la ejecución de esa conducta. Para Ayllon \& Azrin (1974) la saciedad fija un límite sobre un número de fichas que se pueden dar por una tarea, el cual no puede ser tan grande que permita al individuo pasársela largo tiempo sin ganar fichas, viviendo solamente de las que ganó anteriormente; por tanto, toda conducta se espera en un tiempo y lugar determinado y el reforzador debe tener los dos aspectos en cuenta, asegurándose que quien entrega los reforzadores esté siempre disponible para hacerlo. De no ser así, se altera la efectividad del tratamiento.

Es preciso aclarar que para que este sistema funcione de manera adecuada, previa y concertadamente se fija el valor de intercambio de las fichas que se otorguen y las conductas que van a ser premiadas y adoptadas por el sujeto. El programa refuerza con fichas la conducta deseada (cada vez que ella ocurra), para motivar al sujeto a implementarla; sin embargo, cuando las conductas se consoliden, se deben espaciar los intervalos de intercambio para lograr una deshabituación progresiva de las fichas y de los reforzadores que obtiene el sujeto y reemplazarlos por reforzadores sociales como elogios y reconocimientos. Para Rimm \& Masters (1980) hay riesgos al terminar abruptamente estos programas, porque mantener los nuevos patrones de conducta depende solamente de las contingencias naturales o de los reforzadores sociales; he allí la importancia de diseñar adecuadamente la fase de retirada del tratamiento para obtener su máximo provecho.

La economía de fichas, a pesar de ser una técnica de fácil aplicación en instituciones psiquiátricas, las experiencias sobre éstas en publicaciones son muy reducidas, más, si se tiene en cuenta que el programa ha demostrado ser exitoso al aplicarse en instituciones penitenciarias, instituciones psiquiátricas, niños con desordenes conductuales y trastornos de la conducta alimentaria, por sólo mencionar algunas. Para evidenciar esto, es preciso citar algunos estudios basados en aplicaciones de la economía de fichas, para tener una idea de la utilidad e importancia del sistema.
Espada, Méndez \& Orgilés (2005) evaluaron la efectividad de esta técnica al tratar la fobia a la oscuridad, examinando la contribución del sistema a la eficacia del programa de escenificaciones emotivas. Se trabajó con 65 niños (con edad media de seis años y tres meses), asignándolos aleatoriamente a tres condiciones experimentales: 1 . Tratamiento con el programa de escenificaciones emotivas con economía de fichas; 2 . Tratamiento con el programa de escenificaciones emotivas sin economía de fichas y 3 . Grupo de control en lista de espera. El tratamiento fue aplicado por los padres, con doce sesiones de 30 minutos de duración, después de haber realizado el respectivo entrenamiento. Se encontraron diferencias significativas entre las tres condiciones experimentales mencionadas. El tratamiento expuesto en la primera condición experimental alcanzó resultados muy superiores al programa sin economía de fichas, al disminuir su temor a la oscuridad significativamente, lo que revela la importancia, eficacia y utilidad del programa. Los niños del grupo de control mostraron conductas de evitación y expresaron un nivel alto de temor a la oscuridad después del periodo de espera, con diferencias significativas frente a los otros tratamientos; por tanto la economía de fichas es útil en el tratamiento de la fobia a la oscuridad en la infancia.

Por su parte, Moreno, Lora \& Guerreiro (2003) probaron el sistema con trastornos en la ingestión alimentaria en un niño de seis años. En primer lugar se llevó a cabo un programa de entrenamiento con los padres para favorecer la adquisición de habilidades en el manejo del sistema de fichas en el hogar, con el fin de modificar conductas problema. Los resultados evidencian cómo los padres desarrollaron eficazmente las habilidades aprendidas, lo que incrementó su capacidad de afrontamiento ante nuevas situaciones problema que se puedan presentar. Con respecto al niño se observó cómo se instauraron hábitos, tales como el comer sólo e ingerir comida sin triturar aumentando así la cantidad y variedad de comida ingerida. En conclusión se evidencia la eficacia del control ambiental aplicado por el sistema cuando se usa en el tratamiento por parte de los padres para enfrentar problemas alimenticios de sus hijos. 
Es importante citar la experiencia de Olivares, Méndez \& Maciá (1990), quienes combinaron la economía de fichas con técnicas de relajación en grupo y desensibilización en un sujeto con retraso mental de catorce años de edad, que mostraba mutismo selectivo como producto de un proceso de aprendizaje. En primer lugar se realizó una fase de evaluación, desarrollando entrevistas con los padres, la profesora y la educadora, acompañado de la aplicación de varios test psicométricos. Se realizó un registro conductual del mutismo selectivo, con uso de viodegrabaciones en el aula de clase, donde se registraba la frecuencia y longitud de la respuesta verbal al finalizar la jornada escolar. Se registró también la conducta de aproximación física en los espacios de recreo, reportada directamente por los observadores, determinando claramente los intervalos de observación. El periodo de línea base se realizó a lo largo de una semana escolar, luego se hizo exploración de los refuerzos (por ejemplo la actividad física en el juego de baloncesto, golosinas y refrescos) y se llevó una intervención en dos fases, de 2 y 5 semanas respectivamente. La relajación en grupo se hizo quince minutos antes de la sesión de desensibilización sistemática en vivo y el sistema de fichas se estableció por medio de un contrato del que fue informada la clase, acompañado de una hoja de registro en el que se anotaban las fichas obtenidas, ya que los reforzadores que se entregaron beneficiaron tanto al sujeto como a la clase. El inicio del tratamiento produce inmediatamente un rápido incremento del número de verbalizaciones en el sujeto, llegando a alcanzar 20 a la hora y 14 al final de la primera fase del tratamiento. La longitud de las respuestas verbales pasó de dos a cien palabras. De igual forma la conducta de permanecer a menos de un metro de distancia de otro individuo, aumentó en frecuencia y duración, aunque se instauró en el contrato después del undécimo día de tratamiento.

El programa de refuerzo diseñado incluía distintas fases de razón fija (RF), comenzando a reforzar cada respuesta en una tasa (RF1) -refuerzo positivo y continuo-, para concluir, en una última fase, reforzando cada diez respuestas verbales (RF10). La retirada del programa individual se realizó pasando a un contrato entre clase y profesora, en el que los reforzadores de apoyo fueron mantenidos, pero se reformularon las cláusulas del contrato adaptándolas a conductas de rendimiento académico. Los resultados logrados en la aplicación, se mantuvieron durante el seguimiento, lo que permitió al sujeto una integración efectiva al entorno escolar, y demuestra la efectividad de este paquete de intervención compuesto, en el que se integra el sistema de economía de fichas, con técnicas de relajación y desensibilización en vivo. Sería de interés indagar el aporte de estas dos últimas técnicas en el cambio conductual y especificar si el resultado arrojado es más un producto del fortalecimiento en el ambiente a través del programa.

Otra aplicación interesante de la economía de fichas es la realizada por Peinado (1980) en su tesis doctoral, sobre responsividad de los deficientes mentales a la aplicación del sistema de fichas en la clase. Los participantes fueron ocho clases de retrasados mentales, cuatro experimentales y cuatro de control, con condiciones de retraso profundo, moderado, ligero y límite. En las clases experimentales con la implantación del sistema, se registra responsividad significativa de todas las condiciones de retraso, aunque diferencial según el grado del mismo, denotando la eficacia y utilidad del sistema en el tratamiento de conductas en niños con retraso mental.

Por otro lado, Virués (2004) presenta el caso de un paciente con diagnóstico de trastorno límite de personalidad, institucionalizado en una comunidad terapéutica con historia psiquiátrica y problemas de comportamiento. Inicialmente se procedió a aplicar un análisis funcional exhaustivo de las conductas problema, y posterior al mismo, la intervención consideró como foco de conductas deseables, el control de consumo de estimulantes, aumento de actividades de ocio, aumento de comportamientos de autocuidado y reducción de conductas problema, mediante la economía de fichas y control de contingencias, medidas que fueron implementadas a través de un contrato conductual. Durante los dos meses de vigencia del tratamiento, se observó una dramática reducción de todos los comportamientos problema, así como de otras conductas que no fueron objeto 
directo de intervención; por ello, el sistema de tratamiento permitió un mejoramiento significativo de la calidad de vida del paciente.

Carrasco (2008) hizo una aplicación de un programa de modificación de conducta, basado en economía de fichas, en un hogar para acogida de menores víctimas de maltrato físico y emocional por parte de sus padres, en la ciudad de Guatemala. En primer lugar se realizó la evaluación y análisis funcional, con registro de conductas disruptivas que se clasificaron en tres grupos: ejercicio de autoridad, agresividad física y descarga oposición. Con el tratamiento se buscaba eliminar las conductas disruptivas de los menores, utilizando técnicas de moldeamiento y reforzamiento diferencial de otras conductas (RDO). En los resultados se muestra la validez del programa, ya que las conductas disruptivas de todos los participantes, se redujeron en un $75,56 \%$ terminada la intervención, lo que denota un porcentaje altamente significativo.

Saldaña (1984) comenta ampliamente las aportaciones de la perspectiva conductual al campo de la salud, al nombrar los procedimientos conductuales utilizados en la prevención primaria, secundaria y terciaria. Dentro de tales procedimientos cita la economía de fichas, que se clasifica en los procedimientos de manipulación de contingencias, resaltando su amplia utilidad frente a aspectos tales como los estilos de alimentación y el aumento de la actividad física en el nivel primario, adherencia médica en el nivel secundario y control de dosis de insulina en diabéticos a nivel terciario, que permite fomentar el aprendizaje de habilidades para controlar la enfermedad.

En el contexto colombiano es importante mencionar la experiencia de Ospina (2003), quien desarrolló un programa de economía de fichas en niños, con trastorno deficitario de la atención e hiperactividad asociado a su discapacidad intelectual en niños y niñas de la Fundación Cindes de la ciudad de Pereira. El sistema se basó en actividades recreativas como la musicoterapia, actividad acuática terapéutica, artes manuales, sistemas y expresión lingüística. En primer lugar se seleccionaron ocho niños diagnosticados con el trastorno, utilizando como forma de medición la observación y los registros conductuales. Luego se establecieron horarios de trabajo en cada una de las actividades propuestas, al utilizar fichas como reforzadores en la emisión de las conductas previamente acordadas en cada área: primero se hizo entrega gratuita de fichas, que correspondían a bonos marcados y firmados al respaldo por el profesional para reconocerlas como objeto de intercambio de reforzadores; luego se entregaron las fichas sólo frente a las conductas deseadas y la entrega de las mismas estuvo a cargo de varias personas.

Una vez establecidas las conductas se realizó una retirada gradual. El programa en esta primera publicación se encontraba en ejecución y se hace un reporte de las dos primeras fases, con una duración de diez semanas, en las cuales se han obtenido logros a nivel comportamental y en lo que refiere al manejo de la atención, permitiendo dirigir y seleccionar la actividad mental en tareas de constante movimiento o aquellas que demandan mayor concentración y atención con aumento del tiempo de permanencia en las actividades. Se incrementó la frecuencia de la puntualidad para ingresar a las actividades, el contacto visual sostenido mientras hay comunicación verbal, la preparación del material y el salón para cada actividad, el saludar y despedirse, permanecer en el salón durante toda la actividad, permanecer sentado durante cinco minutos. Todo ello a través de la realización de actividades de tipo recreativo permitiendo el establecimiento de comportamientos adaptativos.

Como se ha visto, el programa de economía de fichas es de gran importancia para el tratamiento de conductas problemáticas y es una oportunidad para el mejoramiento de la calidad de vida de poblaciones que se encuentran en situaciones en las cuales resulta difícil llegar a cumplir incluso con las habilidades básicas que refieren al autocuidado. Un ejemplo de esto corresponde a la experiencia de Sanjurjo (SF), quien trabajó con personas con trastornos mentales severos y graves, sintomatología negativa y largo tiempo de evolución, en la que había deterioro de sus actividades de la vida diaria, lo cual implicaba la pérdida de 
su autonomía y el aumento de su discapacidad y lo que no permite alcanzar objetivos más avanzados dentro de su plan individualizado de rehabilitación. Se diseñó una intervención, aplicando la técnica de economía de fichas y un programa de terapia ocupacional, con el fin de consolidar la recuperación de las actividades de la vida diaria. Se administra el programa a un paciente de 37 años, diagnosticado con psicosis esquizofrénica residual, alucinaciones auditivas, vivencias de despersonalización, sensación de control de pensamiento e interpretaciones delirantes de tipo persecutorio y heteroagresividad hacia la familia, que acude a un Centro de Rehabilitación de salud mental ambulatorio.

En primer lugar se identificaron las conductas a reforzar que permitieran aumentar su autonomía y que las pudiera mantener en el ambiente natural, tales como hacer la cama, separar la ropa sucia, ordenar la cocina, la ropa limpia, no tener ropa en el suelo, fregar platos y útiles de cocina, limpiar la nevera, no tener comida caducada, entre otras. Se trataba de conductas de nueva adquisición y otras las que se buscaba aumentar su frecuencia; cada una de estas conductas obtuvo su puntuación en el sistema. Para estas conductas de nueva adquisición se realizó entrenamiento conductual basado en instrucciones, modelado y moldeado con reforzamiento verbal. Luego se identificaron los reforzadores de apoyo significativos, como la compra de teléfono móvil, recargas de teléfono, compra de revistas de divulgación científica, compra de cometas, salidas lúdicas, comidas fuera de casa (restaurante). Luego se establecieron sistemas de registro donde se anotaban los puntos obtenidos semanalmente. Se desarrolló un contrato terapéutico para especificar los refuerzos y las conductas objetivo, estableciendo como duración inicial 19 semanas. Se desarrolló un sistema de seguimiento y administración de refuerzos, con un registro de dos veces por semana en donde el mismo paciente calculaba y anotaba sus puntos. En los resultados de esta experiencia se registra entonces, la instauración de hábitos no adquiridos en el paciente y si bien se parte de un nivel muy bajo en la primera semana, una vez iniciado el plan, aumentaron sus conductas ob- jetivo hasta lograr un rendimiento en las últimas semanas muy superior.

Otra experiencia interesante es la de Acevedo et al. (2006) quienes trabajaron con una adolescente de trece años que presentaba trastornos en el patrón de sueño, para que permaneciera en su cuarto durante la noche entera, con la aplicación de un programa de economía de fichas. Se manejó un diseño $A B C$. En la fase $A$, durante seis días se llevó un autoregistro para determinar las horas durante las que permanecía en su habitación. Después de la línea de base, en la fase B, se determinaron límites en cuanto a la hora, para que pudiera salir de su habitación y así recibir reforzamiento. En la última fase, la conducta consistía en permanecer toda la noche en su cuarto. La conducta deseada aumentó significativamente a medida que avanzaban las sesiones, llegando a permanecer en su cuarto durante toda la noche; se reforzó el comportamiento intermitentemente durante aproximadamente dos meses para lograr un cambio permanente y evitar recaídas, lo que corrobora cómo la economía de fichas es aplicable en escenarios naturales como el hogar, con resultados favorables.

LePage (1999) comenta la experiencia de investigación de un sistema de economía de fichas introducido en una unidad psiquiátrica de cuidado agudo de un hospital rural y su impacto en las lesiones y eventos negativos que suceden cotidianamente entre los internos. Los eventos negativos se definieron como aquellos en que el paciente ejecutaba lesiones que no eran accidentales contra otros, al igual que el no uso de medicamentos de emergencia, y fueron calculados en periodos de dos y cuatro meses antes y después de la implementación de la economía de fichas, sobre 24 pacientes internos en la unidad de cuidado agudo de la casa del Nuevo Hospital de Adultos, con población entre 18 y 20 años. Se elaboró un análisis de covarianza en el que se reporta cómo el número de eventos negativos disminuyó significativamente después de la instauración del sistema, de 129 eventos en los 4 meses anteriores a la implementación, a 73 después del inicio del tratamiento, lo que denota un $43 \%$ de reducción en 
lesiones personales y eventos negativos; también se reporta un pequeño incremento en el uso de medicamentos de emergencia aunque este no fue estadísticamente significativo. En esta investigación se encuentra soporte para el uso de la economía de fichas en escenarios psiquiátricos agudos, con un alto impacto como tecnología para facilitar el cambio.

Aguilar \& Navarro (2008) utilizaron la técnica conductual de economía de fichas con tres estudiantes bajo tutela judicial, que presentaban problemas de adaptación escolar, negándose a desarrollar las tareas al hacer ruidos inadecuados con la boca, arruinar el material escolar, utilizar agresiones verbales a sus pares y educadores, y en general falta de motivación frente a las actividades académicas. En primer lugar se hizo un estudio conductual de cada uno, estableciendo las conductas objeto de intervención según la situación particular de cada sujeto, como por ejemplo, eliminar o reducir los ruidos inoportunos, eliminar o reducir los "maullidos", reducir los comentarios verbales de oposición a realizar las tareas escolares, entre otros. Luego se llevó a cabo una evaluación de las preferencias de los estímulos, cuando se presentaron al menos cinco pares de reforzadores diferentes a cada participante, para que eligieran el que más les interesaba. Algunos ejemplos son: obtener atención del profesor, la calificación positiva de su trabajo escolar, informar positivamente a su tutor del centro de internamiento, ayuda en la realización de tareas, entre otros. Se hizo uso del contrato de contingencias que iba modificándose según el caso particular en dos de los casos, y en el tercero, se aplicó la economía de fichas, con cartulinas y reforzadores de apoyo, acumulando puntos por cada conducta deseable. Tras la intervención se mejoró la adaptación escolar y disminuyeron significativamente las conductas disruptivas. En el participante que se aplicó el sistema de fichas, se redujeron el número de quejas durante las sesiones de trabajo y aumentaron significativamente las tareas escolares en que tenía una participación activa. En conclusión, el sistema de refuerzos permitió una mejor actitud frente a las actividades escolares.
Es importante mencionar que también se han estudiado experimentalmente los efectos de la administración de refuerzo social y de refuerzo material con fichas sobre las conductas verbales, intraverbales y textuales en un programa de aprendizaje para niños con deficiencia auditiva profunda, en el Centro Municipal Fonoaudiológico de Barcelona. Se ha demostrado la superioridad del refuerzo con fichas sobre el refuerzo social en el aprendizaje de las tareas planteadas. El refuerzo material ha actuado como un potente elemento motivacional y de feed-back corrector, siendo más poderoso que el social (Arnau, Calvet \& Sanuy, 1981).

De igual forma se han desarrollado modelos de intervención multimodales en procesos de aplicación y comprobación, en donde se integra el sistema de economía de fichas con otros procedimientos, tal como ocurre con Moreno (2001) quien articula procedimientos de manejo de contingencias y técnicas cognitivas, como reforzamiento, control de estímulos, economía de fichas, tiempo fuera y entrenamiento en autoinstrucciones, en seis etapas sucesivas, aplicando el sistema de fichas en la etapa de los procedimientos de manejo de contingencias y en el que se busca reducir la conducta de hiperactividad que interfiere con el aprendizaje y aumentar el rendimiento académico, ofreciendo un programa terapéutico completo al respecto.

Stover (2004) refiere haber utilizado la economía de fichas junto con costo de respuesta para disminuir verbalizaciones inapropiadas en estudiantes de séptimo y octavo grado; Mateos y Solano (2004) lo utilizaron exitosamente en el tratamiento de la anorexia y bulimia nerviosa. Hail (2004) fue un sistema exitoso para establecer la toma de decisiones financiera en estudiantes de quinto grado, Anderson y Katsiyannis (2004) lo utilizaron para establecer conductas de disciplina en estudiantes de quinto grado con problemas de comportamiento y Gannon, Harmon y Williams (2004) reportan su utilidad en escenarios naturales como el hogar (citados en Acevedo \& cols, 2006).

La economía de fichas ha sido ampliamente utilizada como una alternativa de tratamiento, re- 
habilitación y educación a nivel comportamental, en el que se incluye una importante escala de programas de evaluación y comparación, aplicada en una gran variedad de rangos de población, escenarios y problemas y con la obtención de resultados favorables de acuerdo con sus principios fundamentales. Dentro de este marco, temas novedosos han llegado a ser objeto de discusión, tales como, la integración de la economía de fichas en los escenarios institucionales a pesar de las restricciones que comúnmente se presentan. Las demandas para mantener la integridad del tratamiento y la diseminación de sus procedimientos a mayor escala son temas objeto de las investigaciones actuales en la última década, con progresos considerables, al superar los cuestionamientos de décadas anteriores que se referían a los obstáculos que se presentan y lograr con ello una efectiva aplicación del procedimiento, tales como la identificación de estrategias para mejorar la eficacia del programa que lleven a un incremento de las respuestas del sujeto, el entrenamiento efectivo del personal administrador del sistema, la superación de la resistencia del cliente al programa y la promoción del mantenimiento de la conducta, asegurando la generalización y la transferencia del tratamiento. Al respecto, es claro que ha habido un aumento en el número de estudios, al comparar la economía de fichas con otras intervenciones, en el que se registran importantes avances en estas áreas, cuestión que ha permitido el perfeccionamiento de la técnica, al reconocer de manera clara las variables que pueden contribuir a su eficacia (Kazdin, 1982).

\section{Método}

\section{Participantes}

El programa de economía de fichas fue aplicado a un niño de sexo masculino, de siete años de edad, estudiante de primero de primaria y quien vive en Bogotá. El niño llega al servicio de psicología porque ha tenido problemas escolares, tanto así, que está repitiendo el primer año de primaria en un colegio nuevo. Se pudo establecer que la separación de sus padres ha sido un incidente que lo ha afectado conductualmente en sus emociones y en su desempeño escolar y casero. Convivió un tiempo con su madre, pero al mantener una relación conflictiva con ella (caracterizada por agresiones físicas y verbales constantes), se traslada a la ciudad de Bogotá, en donde vive (hasta el momento) con su padre, tías y abuela. En el colegio ha obtenido algunos llamados de atención, por parte de los profesores, ya que el niño en ocasiones contesta de forma grosera a las personas que le rodean, y hace continuamente pataletas, lo que es evidente en el hogar. Mientras permanece en la casa, gran parte del día lo ocupa en ver TV, no ordena sus juguetes, o su ropa, ni tiende su cama y por tanto el aspecto de su cuarto es desordenado.

En cuanto a su cuidado personal, en términos de conductas, como: peinarse, vestirse solo, cepillarse los dientes, se constituyen en hábitos, que ya según su edad se presentan sin problema, pero hay gran resistencia en bañarse solo, por lo cual genera pataletas y molestias, también, por tener que desarrollar sus labores escolares, o porque en su defecto, en el ejercicio de las mismas, se le hace una crítica constructiva, para su mejoramiento. En general, es muy resistente en el proceso de seguimiento de instrucciones o llamados, a ejecutar conductas como almorzar, al arreglo de su entorno, específicamente, de su cuarto, lo que desencadena en frecuentes llamados de atención, que en ocasiones no son respondidos y por lo cual las labores son finalmente ejecutadas por la abuela. En general, se encuentra en proceso de adaptación a su nuevo hogar, pero su conducta en ocasiones es desordenada y difícil.

Antes de instaurar el programa, se contó con el consentimiento del padre y de los adultos responsables de su cuidado, se realizaron las entrevistas preliminares correspondientes. Se hizo una observación constante de su comportamiento, para determinar aquellas conductas que resultaban deseables, al igual que las indeseables, con el fin de instaurar el sistema. Producto de esto se encontró que el niño tiene problemas con el seguimiento de instrucciones, ya que sus padres le deben pedir varias veces que realice alguna actividad y no lo hace de manera inmediata. Algunas de las conductas asociadas al seguimiento de instrucciones son: hacer las tareas que dejan del colegio 
diariamente, tender su cama, bañarse, levantar y ordenar sus juguetes, levantar y ordenar su ropa, almorzar, e ir a misa los domingos. Adicionalmente, vemos que contesta de manera inadecuada y en algunas ocasiones hace pataletas.

\section{Instrumentos}

Para el desarrollo del programa de economía de fichas se utilizaron formatos de registro de cada una de las conductas, en las que se toma nota de la frecuencia con que se presenta cada una de las conductas y en algunos casos de registro también tiempo de ejecución de las mismas. Para la fase de retirada del programa se tuvieron en cuenta los tiempos de ejecución, en unidades de segundos. Asimismo se utilizaron hojas de control de las fichas, por cada semana de tratamiento, en las que se establece el sistema de precios y los reforzadores disponibles, al igual que los saldos correspondientes. En cuanto a los reforzadores, se utilizaron cerca de 28 , que en su mayoría incluyen eventos durables como: cuentos infantiles, juguetes para niños, algunas golosinas (chocolatinas, helados, yogures), comidas rápidas y reforzadores que consisten en ciertas actividades, como: sesiones de cine casero, acceso a la TV, las cuales proporcionan una variedad de fuentes de reforzamiento. Se utilizaron 80 fichas durables de madera con figuras de diferentes colores, que sirvieron como reforzadores condicionados de cada conducta y dos cajas de plástico, una para el niño, y otra para los administradores del sistema de acceso restringido, para almacenar las fichas.

\section{Diseño}

Se empleó un diseño $A B A$, que consistió en tomar una línea de base de las conductas deseables e indeseables previamente especificadas, por un tiempo de tres semanas (A). Posteriormente se ejecutó el tratamiento, cuyo objetivo era hacer una aplicación controlada de un Programa de Economía de Fichas en el hogar, con el objetivo de lograr un mejor ajuste del comportamiento del niño al ámbito escolar y familiar durante cuatro semanas (B) y finalmente se retiró el tratamiento, registrando la ocurrencia de las conductas con el fin de observar la estabilidad de las mismas, en ausencia del tratamiento, y cómo se mantenían en el ambiente natural, durante un periodo de dos semanas.

\section{Procedimiento}

Se instauró un sistema de economía de fichas siguiendo el modelo descrito por Ayllon \& Azrin (1974). Se utilizaron el reforzamiento positivo para promover la ocurrencia de conductas deseables y el costo de respuesta para propiciar el decremento de las conductas indeseables. Como conductas deseables se seleccionaron las asociadas con el seguimiento de instrucciones: hacer los deberes escolares diariamente, tender la cama, bañarse, levantar y ordenar sus juguetes, levantar y ordenar su ropa, almorzar, e ir a misa los domingos. Para estas conductas no sólo se tuvo en cuenta el criterio de ocurrencia, sino que dicha conducta se presentará de forma completa (como el caso de hacer tareas). De este modo, se consideran como conductas indeseables, el hacer pataletas y contestar inadecuadamente, si para el caso, no se lleva a cabo la tarea. Conductas tales como tender la cama, levantar y ordenar la ropa, debieron ser moldeadas, ya que no se estaban dando de manera adecuada, por lo cual se reforzaron acercamientos sucesivos, partiendo de las conductas que ya presentaba el niño. Los administradores del sistema fueron la abuela y la tía del niño a quienes se entrenaron para este propósito.

Se le dio un precio a cada una de las conductas, de acuerdo con el nivel de importancia que tenían. El costo de los reforzadores se asignó de acuerdo con el grado de preferencia del niño. El sistema estuvo todo el tiempo sujeto a reajuste, sobre todo en la primera semana, para evitar que se acumularan fichas, asegurando siempre que el niño tuviera la posibilidad de alcanzar los reforzadores. Este ajuste se realizó con el fin de mantener el equilibrio económico del sistema. En la primera semana (en el caso de aquellas conductas incluidas en la dimensión de seguir instrucciones), sólo se reforzaban sí y solo sí, la ejecución deseable se iniciaba en un periodo de tres minutos después de dada la instrucción; 
en la segunda semana, dos minutos después; en la tercera semana, un minuto y en la última del tratamiento se entregaba la ficha si la conducta ocurría hasta máximo 30 segundos después de dada la instrucción. Sólo se realizaba un llamado ante cada conducta, teniendo la precaución de evitar dar instrucciones contradictorias por parte de las dos personas que permanecían en el hogar; los reforzadores de apoyo eran entregados en el mismo lugar, siempre y cuando el niño tuviera las fichas necesarias para disponer de los mismos e igualmente eran entregados por varias personas. En la tercera y cuarta semana, se hizo una baja de precios, y la posibilidad de acceso a cinco nuevos reforzadores.

Al inicio de la intervención se explicó claramente al niño, las conductas deseables por lograr y por las que se otorgarán fichas y las indeseables a multar. También se mostraron cada uno de los privilegios que se obtendrían mediante el canje de fichas, haciendo así una muestra de cada uno de los reforzadores, para asegurar que si no se usaban, no sería por falta de familiaridad. La hoja de control y los reforzadores permanecían todo el tiempo a la vista del niño. Se otorgaron y quitaron las fichas inmediatamente después de ocurridas las conductas e igualmente no se permitió que el niño acumulara más fichas de las que podía gastar en un periodo corto de tiempo, de tal forma que el sistema siempre mantuvo su equilibrio y nunca se quedó sin fichas. Durante la transición de la primera a la segunda semana, se hizo un cambio de precios en dos conductas: hacer sus tareas al primer llamado y bañarse, donde se paso de dar tres fichas a dos fichas, ya que se estaban acumulando fichas diariamente, y se estaba perturbando el equilibrio. El resto de valores permanecieron estables por todo el tratamiento. Nunca se dio crédito, y el niño debió pagar lo instaurado por el sistema de precios antes de adquirir el reforzador. Se controló el ambiente, de tal forma que no pudiera acceder a los reforzadores de una manera diferente a la permitida por el sistema; por ejemplo, el ver televisión sólo era posible a través de las fichas.

Para ayudar con el equilibrio del sistema se reforzaron conductas que ya estaban instauradas en el repertorio conductual del niño, sin necesidad de dar la orden como cepillarse los dientes, peinarse y vestirse solo. Siempre que se entregaban las fichas, se explicaba al niño porqué la obtenía y se acompañaba de reforzadores verbales. Es importante resaltar que las conductas que se han determinado aquí como deseables o indeseables se han identificado luego de haber realizado observaciones del comportamiento y su impacto en el medio: específicamente en las relaciones con los padres y la abuela y viceversa.

\section{Resultados}

Las figuras muestran la tendencia de cada una de las conductas en la línea de base, la implementación del programa y el desvanecimiento del mismo semanalmente. Para las conductas asociadas al seguimiento de instrucciones, el criterio de cambio conductual estuvo determinado por el decremento en el número de veces que es necesario dar la instrucción para que ella sea ejecutada por parte del niño. En la fase de implementación del programa de economía de fichas, sólo se entregaba el reforzador si la conducta ocurría una vez dada la instrucción. En esta fase se pidió a los administradores del sistema dar la instrucción una sola vez. Como las figuras muestran el registro semanal, el mínimo de instrucciones a la semana para la ejecución de la conducta es de siete (7), que corresponde a una instrucción diaria.

La Figura 1 muestra el número de instrucciones que era necesario dar al niño antes de la implementación del programa en la que se solicitaba que empezara a hacer los deberes escolares. Apenas se inicia el programa ya se observa un decremento en el número de instrucciones, llegando a darse solamente una instrucción diaria. El registro se hacía una vez ocurriera la conducta después de dada la instrucción. Desde que se empezó la implementación del programa, se dio un tiempo de 3 minutos (en la semana 4), 2 minutos (semana 5), un minuto (semana 6), 30 segundos (semana 7) para que la conducta empezara a ocurrir. 


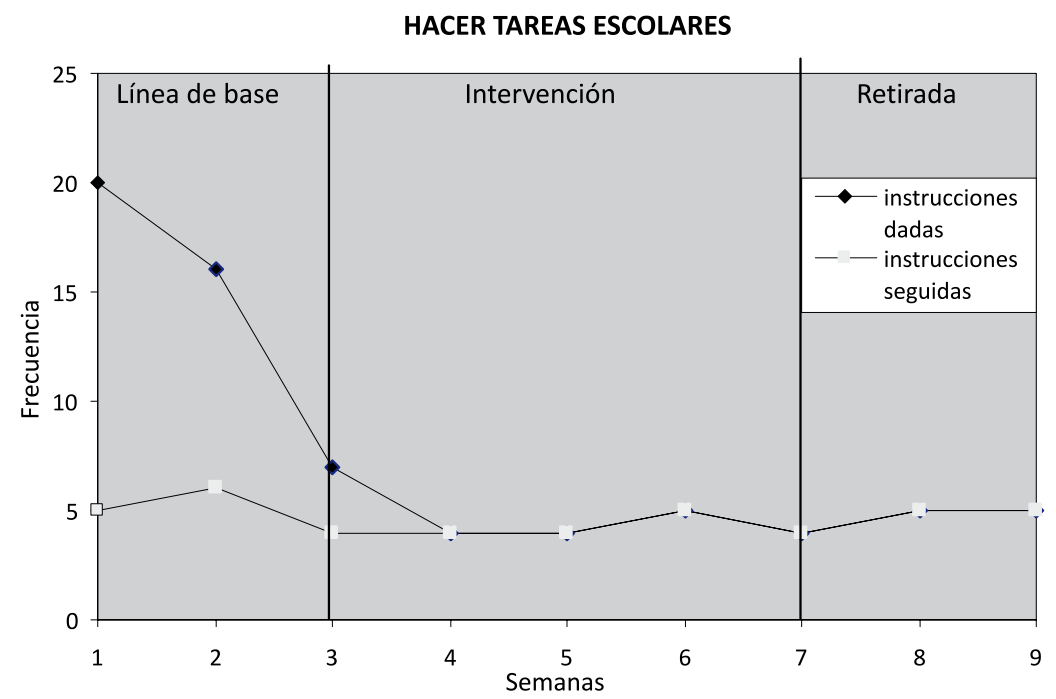

Figura 1. Instrucciones dadas y seguidas para hacer tareas

La figura no sólo muestra el decremento en el número de instrucciones, sino que además hay un decremento en la latencia, lo que es bastante positivo. En la fase de retirada del programa se observa un mantenimiento en el número de instrucciones dadas al respecto. En la línea de base muchas de las peticiones de hacer tareas no eran seguidas, es decir, en muchas ocasiones el niño no hacía las tareas. La figura no muestra un incremento en el número de veces que se hace tareas, sino el ajuste perfecto entre la petición y el cumplimiento de la misma.

La Figura 2 muestra el número de instrucciones dadas para que el niño hiciera la cama versus el número de veces en que realmente lo hizo. Hay que tener en cuenta que a esta conducta fue necesario moldearla, ya que si bien ocurría de alguna forma, la topografía de la misma no era la más adecuada. De nuevo, la implementación del programa favoreció la reducción en el número de veces que se solicitaba por parte de los administradores del programa el arreglo de la cama. Decremento que se mantiene en la fase de retirada. También se observa, respecto de la línea de base, un incremento en el número de veces que el niño tiende la cama; tendencia que se mantiene en la fase de retirada del programa. En esta fase, incluso la conducta llegó a presentarse sin haber dado la instrucción.

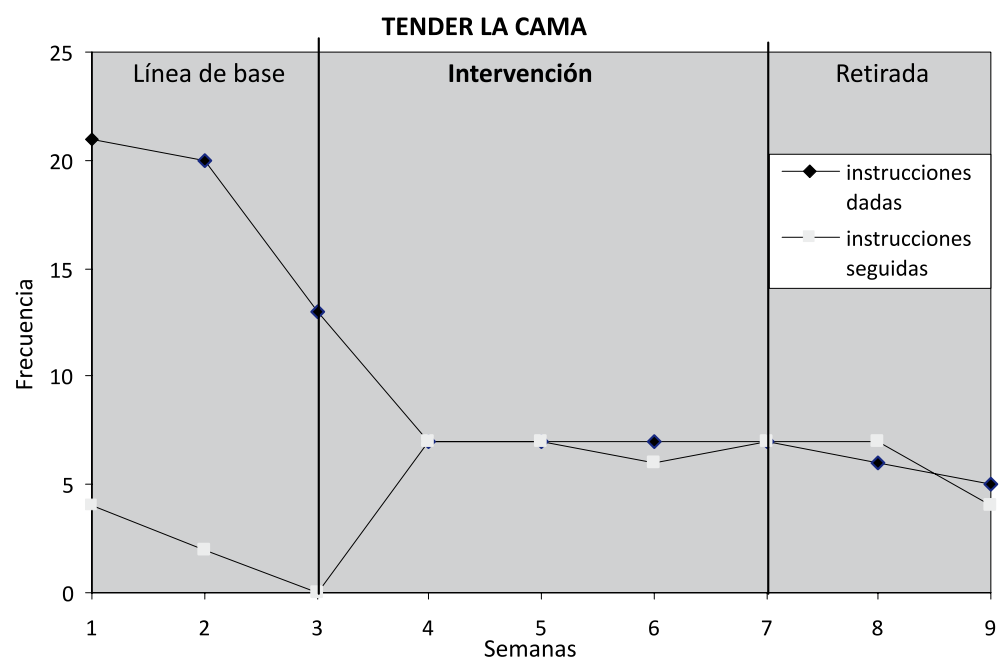

Figura 2. Número de instrucciones dadas y seguidas para arreglar la cama 
La Figura 3 muestra también el decremento en el número de veces que se solicitaba al niño que se bañara, si se tiene en cuenta la fase de intervención respecto de la línea de base. Esta conducta al inicio del programa era una de las que menos probabilidad de ocurrencia tenía, ya que se hacían muchas peticiones sin obtener resultado alguno. En la fase de implementación se decrementó el número de instrucciones dadas y si bien la conducta sí ocurría una vez dada la instrucción; dicha ejecución no se ajustaba al criterio de cumplimiento luego de los 3 minutos, 2 minutos, el minuto o 30 segundos. Sin embargo, sí se observó un decremento en la latencia. En la fase de retirada el decremento en el número de peticiones se mantuvo, pero el cumplimiento de la misma no se ajustó a los criterios de cumplimiento.

La Figura 4 muestra la forma como ocurría la conducta de levantar y ordenar los juguetes. Se hacían muchas peticiones al respeto en la fase de línea de base y una vez se empezó la aplicación del programa e incluso en la fase de retirada del mismo se mantuvo en las 7 peticiones semanales, es decir, una petición diaria. Al igual que las anteriores, cada semana del programa se decrementaba el tiempo que se daba para que la conducta se empezara a dar. Respecto de la ocurrencia de la conducta, se observa en línea de base una probabilidad escasa de ocurrencia y en la fase de implementación se incrementó ajustándose a los criterios de ocurrencia.

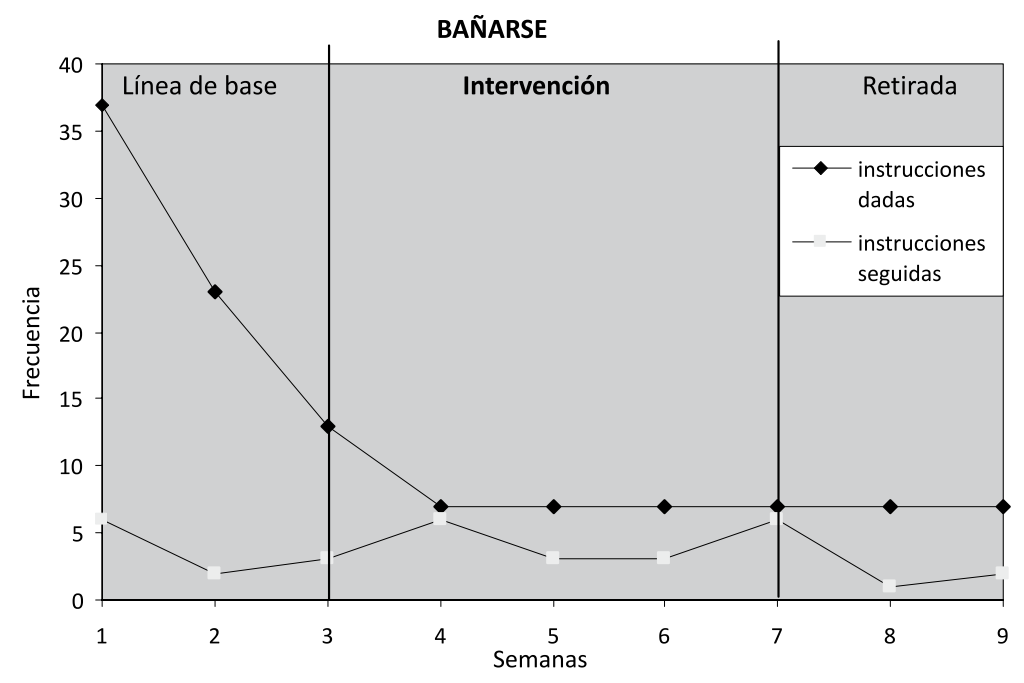

Figura 3. Número de instrucciones dadas y seguidas para bañarse

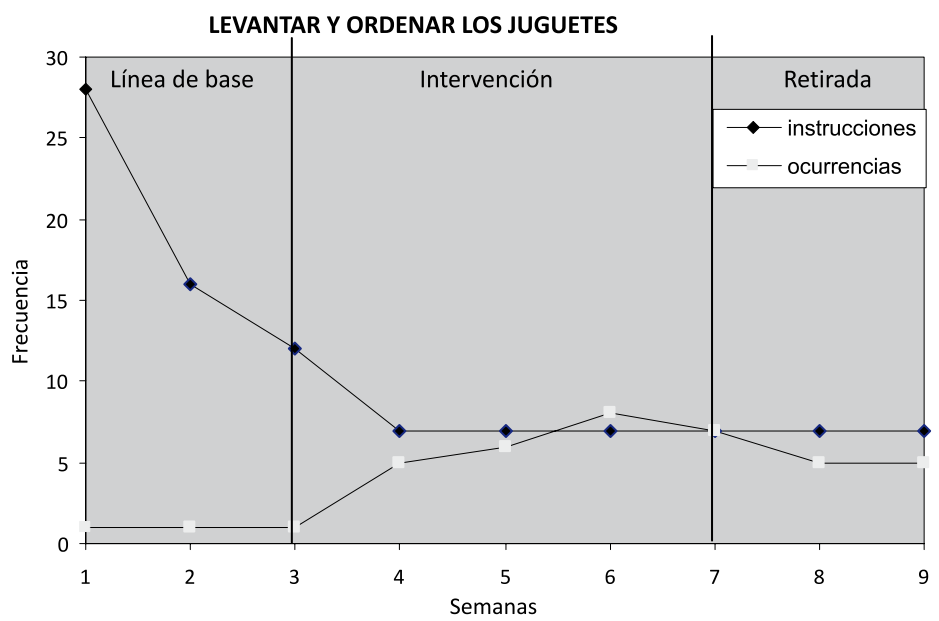

Figura 4. Número de instrucciones dadas y seguidas para levantar y ordenar los juguetes 
En la misma fase incluso llegó a ocurrir la conducta sin que se diera la instrucción. En la fase de retirada la conducta mostró un leve decremento pero aún así se mantuvo por encima de lo mostrado en línea de base.

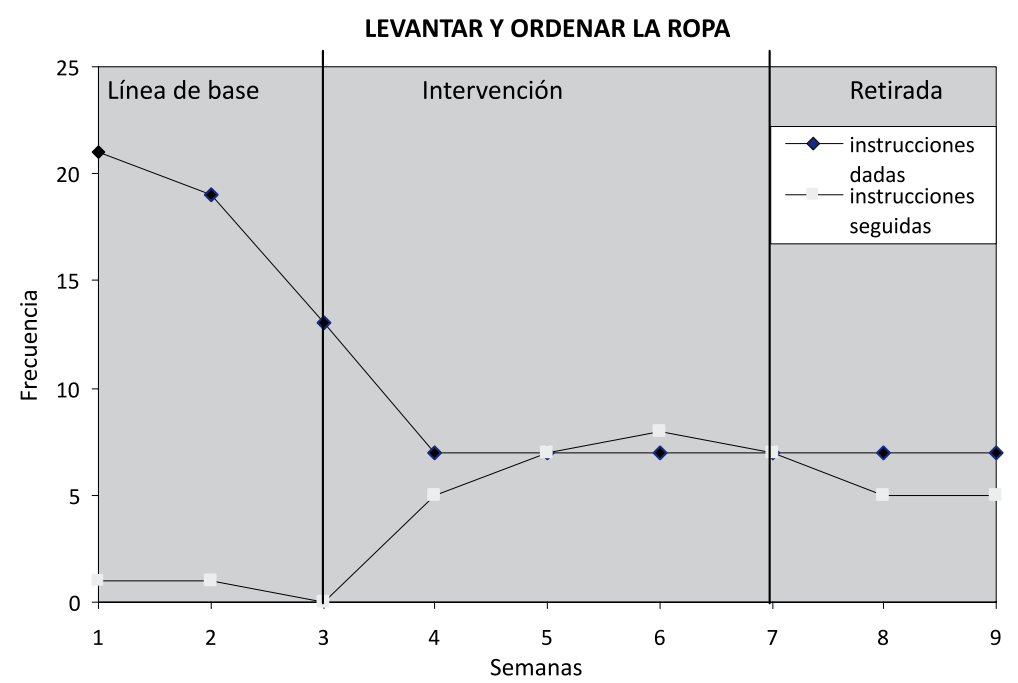

Figura 5. Número de instrucciones dadas y seguidas para levantar y ordenar la ropa

La Figura 5 muestra el número de peticiones que había que hacer al niño para que recogiera y ordenara su ropa. Al igual que las anteriores se presentó un decremento en el número de peticiones que había que hacer al respeto y en la fase de retirada se mantuvo de la misma forma. El criterio para el inicio de la conducta una vez dada la instrucción fue el mismo que para las anteriores. En esta conducta también se evidenció un incremento sustancial entre su frecuencia en línea de base y en fase de implementación y de retirada.
La Figura 6 muestra la misma tendencia en la reducción del número de veces en el que se le pide al niño que almuerce en comparación con las veces en la que ocurre. También se observa un incremento y sobretodo mayor estabilidad en el número de veces que lo hace dentro de la latencia permitida, después de haberle hecho una sola petición.

La Figura 7 muestra lo ocurrido con la asistencia a misa, en la que a pesar de lograrse un decremento en el número de peticiones hechas no se observa cambio en la ocurrencia de este comportamiento.

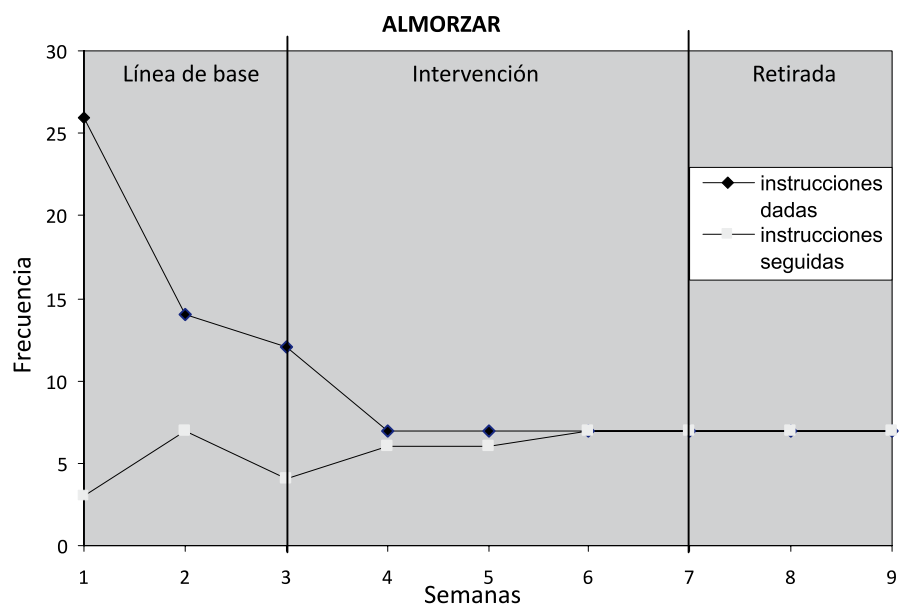

Figura 6. Número de instrucciones dadas y seguidas para almorzar 


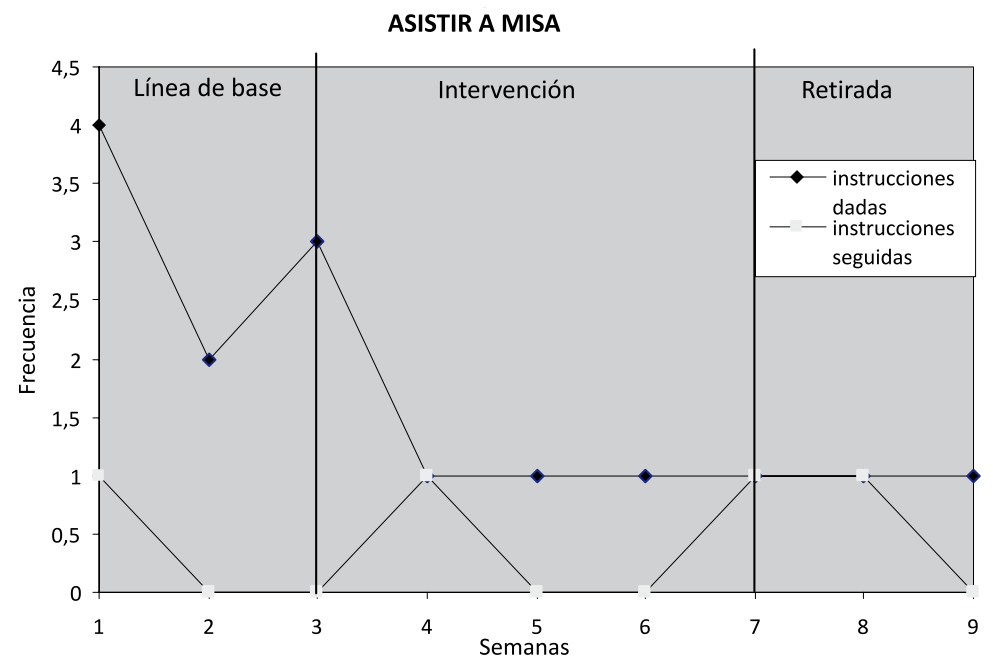

Figura 7. Número de instrucciones dadas y seguidas para asistir a misa

La Figura 8 muestra la tendencia de la conducta de hacer pataletas. Como se observa, hay un decre-

mento notorio en la fase de implementación del programa de economía y al retirarlo la tendencia se mantuvo.

Por último, la Figura 9 representa lo ocurrido cuando se implementó el programa con la con- ducta de contestar de manera inadecuada respecto de la línea de base. Al retirar el sistema la conducta refleja un incremento, lo que permitiría pensar la sensibilidad de esta conducta al sistema de reforzamiento implementado.

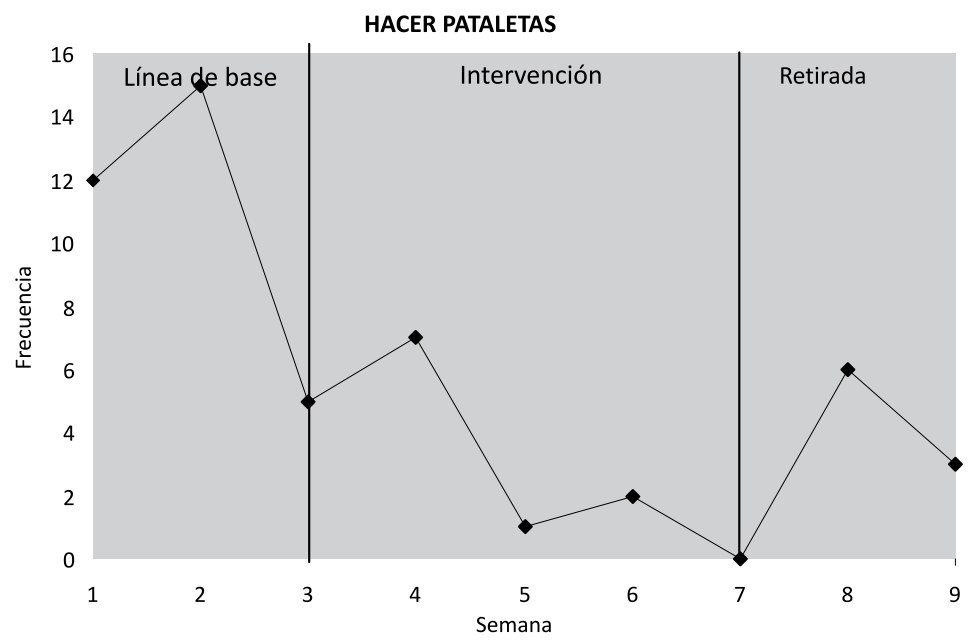

Figura 8. Tendencia de la conducta de hacer pataletas 


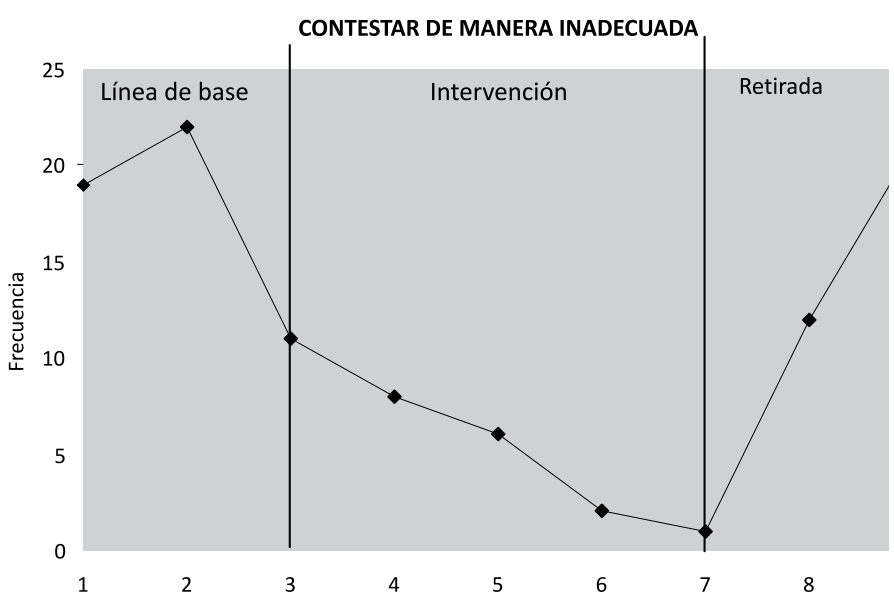

Figura 9. Tendencia de la conducta de contestar de manera inadecuada

\section{Discusión}

Antes de implementar el sistema de economía de fichas, se hicieron los análisis funcionales relacionados con las conductas problemas del niño, por lo que la decisión de utilizar el programa fue producto inicialmente de dichos análisis y los resultados obtenidos ratifican esto.

Como se pudo observar, la implementación del programa fue lo suficientemente exitosa como para garantizar un mejor ajuste del comportamiento del niño a su entorno académico y familiar. Si bien, el caso del niño es bastante complejo, el programa contribuyó no sólo al ajuste de su comportamiento, sino también el de los demás miembros de su entorno, ya que situaciones como, por ejemplo, las instrucciones que se daban de manera intensa para reportar resultados, éstos son un reflejo de la forma inadecuada como se expresan estas instrucciones o la ausencia de consecuencias que favorecieran la ocurrencia del comportamiento. Ello implica que a pesar de que el sistema va dirigido al niño, la administración del mismo implica ajustes en la conducta de los administradores y una forma más adecuada de comunicación en la que se reducen los niveles de conflicto, producto del estrés que genera en la familia el comportamiento desobediente del niño.

Uno de los aspectos interesantes es el hecho de que la mayoría de las conductas empezaron a mostrar un cambio desde la tercera semana de observación (línea de base) antes de la im- plementación del programa. En ese momento el niño estaba enterado de que su comportamiento estaba siendo observado, lo que probablemente influyó en este cambio.

Respecto de la implementación del programa se observó que en la segunda semana algunos reforzadores de apoyo no eran fácilmente accesibles para el niño, por lo que fue necesario hacer un ajuste de precios. Dichos ajustes se hicieron a lo largo del programa, de varias maneras: lo importante es que en el sistema no permita la acumulación de reforzadores condicionados por parte del niño ni que se recurra al crédito (cosa que el sistema no permite) porque los reforzadores sean muy costosos. De tal manera, la relación que se dé entre los reforzadores condicionados (fichas) obtenidos por la emisión del comportamiento y el número de fichas que se tienen que dar a cambio de los reforzadores de apoyo, será determinante en la efectividad del programa. Winkler (1980), Kagel \& Winkler (1972) y Winkler (1972) afirman que para que el sistema de economía de fichas sea exitoso, la relación entre ingresos y gastos debe ser de 1 o tender a 1, lo que permite evidenciar el equilibrio económico del sistema. Según esto, un exceso de ingresos (obtención de muchas fichas porque se paga demasiado por cada uno de los comportamientos) llevará a que se pierda motivación para el comportamiento y por ende el programa fracasará; por otro lado, si el precio de los reforzadores de apoyo (gastos) es muy alto (como ocurrió en las primeras dos semanas) el 
comportamiento también se deteriorará, ya que se percibe que ellos son inalcanzables. De tal manera que lo más difícil en la implementación de un programa de este tipo es tratar de mantener el sistema en equilibrio que permita no sólo un beneficio en este caso para el niño sino además que facilite el desvanecimiento del programa, para que los comportamientos vayan quedando bajo refuerzo natural.

En este caso, los ajustes se dieron por la inclusión de nuevos reforzadores de apoyo a bajo precio (por pocas fichas) y un decremento en el precio de los que ya estaban establecidos.

Otro especto a destacar es que la mayoría de las conductas se mantenían sin que el programa estuviera operando, pero en el caso de contestar de manera inadecuada y bañarse se mostraron muy sensibles a la ausencia de reforzamiento, lo que conllevó a que sin el programa se incrementaran. Esto se habría podido evitar haciendo un mejor desvanecimiento del programa (pagando menos fichas por las conductas o incrementando el precio de los reforzadores de apoyo) o prolongando un poco más la duración del mismo.

Es especialmente diciente el que a pesar de que se presentan incrementos en la gran mayoría de las conductas deseables, este incremento se hubiera hecho más evidente si no se hubiera tenido en cuenta el criterio de latencia que se dio al niño y que fue variando semanalmente. En muchos casos en los que el niño no obtuvo los reforzadores previstos, no fue porque el comportamiento no hubiera ocurrido sino porque no ocurría dentro del tiempo en el que debía ocurrir.

Otro aspecto interesante y que no se mostró en las figuras pero que sí se registró, es que también en algunas conductas se observó un decremento en el tiempo que el niño demoraba en ejecutarlas. Por ejemplo, al inicio del programa se podía demorar bastante recogiendo los juguetes o la ropa; la implementación del programa permitió también hacer ajustes en ese sentido, de tal manera que al final muchas de las conductas se daban mucho mas rápido.
La economía de fichas demuestra ser una técnica útil para trabajar en la crianza de los niños y tiene la ventaja adicional de que se pueden trabajar muchas conductas de manera simultánea: al mismo tiempo, prepara mejor a los padres para afrontar de manera más adecuada esta labor, reduciendo el manejo inadecuado de emociones, disminuyendo situaciones de agresividad y maltrato y, algo muy común, previene el uso de criterios diferentes entre los padres, respecto de la educación y formación de los hijos.

\section{Referencias}

Acevedo, A., Cota, C., Helú, P., Tello de Meneses, C., Tena, M. \& Guzmán, J. (2006). Aplicación de economía de fichas en un caso de trastorno en el patrón del sueño. En Revista Enseñanza e investigación en Psicología, 11 (1), 177-184.

Aguilar, C. \& Navarro, J. (2008). Análisis funcional e intervención con economía de fichas y contrato de contingencias en tres casos de conductas disruptivas en el entorno escolar. En Revista Latinoamericana de Psicología, 40 (1), 133-139.

Alvord, J (1974). Economía de fichas. México: Editorial Ciencia de la Conducta.

Arnau, J., Calvet, P. \& Sanuy, J. (1981). Evaluación de los efectos del refuerzo social y del refuerzo con fichas sobre la conducta verbal de niños deficientes auditivos profundos. Anuario de Psicología, 24, 1.

Ayllón, T. \& Azrin, N. (1974). Economía de fichas: un sistema motivacional para la terapia y la rehabilitación. México: Trillas.

Carrasco, N. (2008). Aplicación de un programa de economía de fichas en un hogar de acogida de menores víctimas de maltrato. Revista electrónica de intervención psicosocial y psicología comunitaria, 3, 11-15. Documento descargable de 3: http://dialnet.unirioja.es/ servlet/oaiart?codigo $=2547021$. 
Espada, J., Méndez, C. \& Orgilés, A. (2005). Contribución de la economía de fichas a la eficacia de las escenificaciones emotivas en el tratamiento de la fobia a la oscuridad. Revista de Ansiedad y Estrés, 11 (1), 7-16. Tomado de: http://dialnet.unirioja.es/servlet.

Kagel, J. \& Winkler, R. (1972). Behavioral economics: areas of cooperative research between economics and applied behavioral analysis. Journal of Applied Behavior Analysis, 5 (3), 335-342.

Kazdin, A. (1982). The Token Economy: a decade later. Journal of Applied Behavior Analysis, 15 (3), 431-445.

LePage, J. (1999). The Impact of a Token Economy on Injuries and Negative Eventson an Acute Psychiatric Unit. Psyquiatric Services, 50 (7), 941-944.

Moreno G., Lora, J. \& Guerreiro, A. (2003). Tratamiento de un trastorno de ingestión alimentaria en la infancia mediante entrenamiento a padres: un estudio de caso. (Versión Electrónica), Psicología conductual: Revista internacional de psicología clínica y de la salud, 2 , 371-387. Tomado de: http://dialnet.unirioja. es/servlet/.

Moreno, I. (2001). Tratamiento psicológico de la hiperactividad infantil: un programa de intervención en el ámbito escolar. Revista de psicología general y aplicada, 54 (1), 81-93.

Olivares, J., Montesinos, L. \& Preciado, J. (2005). A 19th century predecessor of the token economy. Journal of Applied Behavior Analysis. 38 (3), 427.

Olivares, J., Méndez, F. \& Maciá, D. (1990). Tratamiento conductual de un caso de mutismo electivo en un sujeto con retraso mental. Anales de Psicología, 6 (1), 59-70.

Ospina, C. (2003). Implementación de un programa de economía de fichas basado en la recreación para el manejo del trastorno deficitario de la atención con hiperactividad (TDAH) en niños y niñas con discapacidad intelectual de la Fundación Cindes de Pereira. Comité de Investigación de Cindes - CIC.

Peinado, M. (1980). Responsividad de los deficientes mentales a la aplicación de un sistema de economía de fichas en la clase. Disertación doctoral, Universidad Complutense de Madrid, España. Tomado de: http://www.cibernetia.com/tesis_es.

Rey, C., \& Acevedo, A. (2005). Análisis bibliométrico de las tesis de pregrado y postgrado realizadas en Bogotá sobre la implementación y evaluación de programas de terapia y modificación del comportamiento. Acta Colombiana de Psicología, 14, 97-111.

Rimm, D. \& Masters, J. (1980). Terapia de la conducta. Editorial Trillas: México.

Roales, J. \& Fernández, A. (1986). La economía de fichas en el tratamiento de pacientes crónicos: el papel del personal institucional. $R e$ vista Latinoamericana de Psicología, 18 (1), 23-41.

Saldaña, C. (1984). Alternativas conductuales a problemas de salud. Anuarios de Psicología 30/31 (1-2). Departamento de Psicología Experimental: Universidad de Barcelona. Tomado de http://www.raco.cat/index.php/AnuarioPsicologia/.

Sanjurjo, G. (SF). Utilización de técnicas de modificación de conducta en terapia ocupacional. Caso prático / clínico. Recuperado de http:// www.aytona.com/portal/download/Arti7.doc.

Virués, J. (2004). Análisis funcional y tratamiento de un paciente con graves problemas de conducta diagnosticado de trastorno límite de la personalidad (versión electrónica), International Journal of Clinical and Health Psychology, 4, 207 - 232. 
Winkler, R. (1972) A theory of equilibrium in token economies. Journal of Abnormal Psychology, 79 (2), 169-173.
Winkler, R. (1980) Behavioral economics, token economics and applied behavior analysis. En J. E. R. Staddon: Limits to action. New York: Academic Press. 